\title{
A PDE FORMULATION FOR VISCOUS MORPHOLOGICAL OPERATORS WITH EXTENSIONS TO INTENSITY-ADAPTIVE OPERATORS
}

\author{
Petros Maragos ${ }^{1}$ and Corinne Vachier ${ }^{2}$ \\ ${ }^{1}$ School of E.C.E., National Technical University of Athens, Athens 15773, Greece. \\ ${ }^{2}$ CMLA, ENS Cachan, CNRS, PRES UniverSud, 61 Av. President Wilson, F-94230 Cachan, FRANCE. \\ maragosdcs.ntua.gr corinne.vachierdcmla.ens-cachan.fr
}

\begin{abstract}
Viscous morphological operators have shown very good performance in regularizing various image analysis tasks such as detection of intensity-varying boundaries and segmentation. This paper presents a novel formulation of viscous morphological operators as solutions of nonlinear partial differential equations (PDEs) of the hyperbolic type with level-varying speed. Efficient numerical algorithms are also developed to solve these PDEs and generate the viscous operations. It also generalizes the viscous operators by studying the class of intensity level-varying operators, of which special cases are intensity adaptive connected operators such as volume openings and viscous reconstruction filters. We present both theoretical aspects and applications of the above ideas.
\end{abstract}

Index Terms - Morphological operations, Adaptive filters, Partial differential equations.

\section{INTRODUCTION}

Viscous morphological operators have shown very good performance in regularizing various image analysis tasks such as detection of intensity-varying boundaries and segmentation $[18,19]$. Theoretical aspects of viscous lattices and viscous connections have been studied in [16]. Standard morphological operators that depend on a structuring element act everywhere on an input image at a predefined fixed scale (size) and all the structures in the image are processed identically whatever their luminance. However, in many applications, two structures of same size but of different luminance (or contrast) may not have the same visual importance and should not be processed identically. For example, in segmentation tasks, fuzzy or noisy contours require a high amount of modeling while precise contours should not be smoothed. Viscous morphological operators $[11,18,19]$ can be seen as filters of locally adaptive activity, which in the aforementioned applications offer a systematic way to choose between a strong or a weak regularization. Specifically, by assuming that points of high luminance are certain while points of low luminance are uncertain (or conversely), the size of the structuring element is defined at each pixel as a function of the local luminance.

The viscous operators may have a considerable computation load since they require a different filtering for each intensity thresholding level and then stacking. In this paper we present a novel and simple to implement method that generates isotropic viscous morphological operators using hyperbolic partial differential equations (PDEs) whose curve speed varies monotonically according to the

P.M.'s work was supported by the Greek GSRT grants IIENE $\Delta$ 2003E $\Delta 554 \& 865$ and the EU FP6-IST project 'ASPI' and NoE 'MUSCLE'. intensity. They stem from the corresponding constant-speed PDEs that model standard flat morphological operations $[3,8]$. These new PDEs offer both an efficient way to implement the viscous filters as well as a continuous dynamic model.

In contrast to the all-discrete approaches that dominated image processing in the recent past, in computer vision since the late 1980's there have been proposed continuous models for several tasks based on PDEs. The discrete part of such approaches comes only in the difference equations (numerical algorithms) that approximate the solution of these PDEs. Motivations for using PDEs include better and more intuitive mathematical modeling, connections with physics, better approximation to the Euclidean geometry of the problem, and existence of efficient numerical algorithms for solving them. Several classes of nonlinear PDEs used in image analysis and vision are based on or related to morphological operations. In the early 1990s three teams of researchers independently published nonlinear PDEs that model the continuous morphological scale-space $[1,3,20]$. Many refinements of the above three works for morphological PDEs followed. Recent reviews can be found in $[6,8]$. Our PDE work in this paper extends the approach in $[3,8]$. In parallel to the development of the above ideas, there have been some advances in curvesurface evolution using level-set methods [12]. Many applications to image processing and computer vision have followed since then. Early examples include the implementation by $[2,14]$ of the morphological PDEs of [3] for continuous-scale dilations and erosions by using the level-set algorithms of curve evolution.

The next section presents the definitions and some examples of viscous morphological operators. Further, we propose their extension and unification into a more general class of morphological operators that are intensity-level adaptive and include various classes of connected filters. Then we present the major contribution of this paper, the PDE formulation of viscous operators, followed by efficient numerical algorithms to solve them and simulation examples.

\section{VISCOUS MORPHOLOGICAL OPERATORS}

The dilation and erosion of a set $X$ by a disk of radius $\lambda$, i.e. the Minkowski addition $X \oplus \lambda B$ and subtraction $X \ominus \lambda B$, are denoted by $\delta_{\lambda}(X)$ and $\varepsilon_{\lambda}(X)$ respectively ( $B$ is the unit disk). Similarly, $\delta_{\lambda}(f)$ and $\varepsilon_{\lambda}(f)$ denote the flat dilation and erosion of a graylevel image $f$ by such a disk.

A graylevel image $f$ can be decomposed into level sets $X_{h}=$ $\{x \in E: f(x) \geq h\}$ and can be reconstructed from them: $f=$ $\bigvee_{h} h \cdot \chi_{h}(f)$. Here, $E$ denotes the image domain space and $\chi_{h}$ is the indicator function of $X_{h}: \forall x \in E, \chi_{h}(x)=1$ if $x \in X_{h}$, else $\chi_{h}(x)=0$.

The standard flat morphological operators commute with thresh- 
olding. Further, they process all level sets identically. For example, the flat dilation and erosion of a function $f: E \rightarrow[0, M]$ satisfy:

$$
\delta_{\lambda}(f)=\bigvee_{h} h \delta_{\lambda}\left[\chi_{h}(f)\right] \text { and } \varepsilon_{\lambda}(f)=\bigvee_{h} h \varepsilon_{\lambda}\left[\chi_{h}(f)\right]
$$

On the contrary, the viscous morphological dilations and erosions proposed in [19] process different level sets at different scales:

$$
\delta_{\lambda}^{v}(f)=\bigvee_{h} h \delta_{\lambda(h)}\left[\chi_{h}(f)\right] \text { and } \varepsilon_{\lambda}^{v}(f)=\bigvee_{h} h \varepsilon_{\lambda(h)}\left[\chi_{h}(f)\right]
$$

In theory, $\lambda(h)$ can be any function of the luminance $h$ assuming values in the range $\left[0, \lambda_{m}\right]$, where $\lambda$ is the maximal scale of an operator applied on any level set. For the two types of viscous operators studied in [19], $\lambda(h)$ is a linear function of $h: \lambda(h)=\lambda_{m}(M-h) / M$ or $\lambda(h)=\lambda_{m} h / M$. In the first case, points of lowest luminance are strongly dilated $\left(\lambda(0)=\lambda_{m}\right)$ while points of highest luminance are left unchanged $(\lambda(M)=0)$, and conversely in the second case. This leads to the definition of two couples of dual viscous erosions and dilations (we set $\lambda_{m}=M$ ):

$$
\begin{array}{lll}
\delta^{v 1}(f)=\bigvee_{h} h \delta_{M-h}\left[\chi_{h}(f)\right] & , & \delta^{v 2}(f)=\bigvee_{h} h \delta_{h}\left[\chi_{h}(f)\right] \\
\varepsilon^{v 1}(f)=\bigvee_{h} h \varepsilon_{M-h} \chi_{h}(f) & , & \varepsilon^{v 2}(f)=\bigvee_{h} h \varepsilon_{h}\left[\chi_{h}(f)\right]
\end{array}
$$

$\varepsilon^{v 1}$ and $\delta^{v 2}$ are dual, $\varepsilon^{v 1}(-)=.-\delta^{v 2}($.$) , but do not define$ an adjunction. Only the couples $\left(\varepsilon^{v 1}, \delta^{v 1}\right)$ and $\left(\varepsilon^{v 2}, \delta^{v 2}\right)$ define adjunctions in the sense of Galois:

$$
\delta^{v i}(f) \leq g \Longleftrightarrow f \leq \varepsilon^{v i}(g), \quad i=1,2
$$

The adjunctions imply that the compositions $\delta^{v 1} \varepsilon^{v 1}, \delta^{v 2} \varepsilon^{v 2}$, $\varepsilon^{v 1} \delta^{v 1}$ and $\varepsilon^{v 2} \delta^{v 2}$ produce openings and closings. Some of these are the viscous openings and closings defined in [18]:

$$
\begin{aligned}
& \gamma^{v 1}=\bigvee_{h} h \gamma_{M-h} \chi_{h} \quad, \quad \varphi^{v 1}=\bigvee_{h} h \varphi_{M-h} \chi_{h} \\
& \gamma^{v 2}=\bigvee_{h} h \gamma_{h} \chi_{h} \quad, \quad \varphi^{v 2}=\bigvee_{h} h \varphi_{h} \chi_{h}
\end{aligned}
$$

where $\gamma_{h}=\delta_{h} \varepsilon_{h}$ and $\varphi_{h}=\varepsilon_{h} \delta_{h}$ are the flat opening and closing by a disk or radius $h$, respectively. While $\left(\gamma^{v 1}, \varphi^{v 2}\right)$ and $\left(\gamma^{v 2}, \varphi^{v 1}\right)$ are couples of dual transforms, only two of the four viscous open/close operators from (5) equal the compositions of some types of viscous dilations and erosions from (3):

$$
\gamma^{v 2}=\delta^{v 2} \varepsilon^{v 2}, \quad \varphi^{v 1}=\varepsilon^{v 1} \delta^{v 1}
$$

The other two compositions yield inequalities:

$$
\gamma^{v 1} \geq \delta^{v 1} \varepsilon^{v 1}, \quad \varphi^{v 2} \leq \varepsilon^{v 2} \delta^{v 2}
$$

Figure 1 illustrates the behavior of a viscous dilation of type 1 and its use for reconnecting thin contours lines. The viscous dilation is compared to the standard dilation. The viscous operator allows to preserve high intensities while dilating points of lowest intensity. Crest lines of the original image are preserved; holes are filled [19].

Alternating sequential filters (ASFs) [15] are useful in image simplification applications. Their viscous extensions are also powerful, as illustrated by the example of Fig. 2. Viscous (ASFs) consist of multiscale viscous open/closings, possibly of the reconstruction type, and can create very useful filtering effects. Figure 2 shows such a viscous reconstruction $A S F$ of type 2, which performs a luminanceadaptive reconstruction ASF at each level:

$$
\mathrm{ASF}_{r e c}^{v 2}(f)=\bigvee_{h} h \cdot \operatorname{Rec}\left[\chi_{h}(f) \mid \varphi_{h} \gamma_{h} \cdots \varphi_{2} \gamma_{2} \varphi_{1} \gamma_{1}\left(\chi_{h}(f)\right)\right]
$$

where $\operatorname{Rec}(A \mid B)$ is a binary reconstruction filter yielding $A$ if $A \cap$ $B \neq \emptyset$, else $\emptyset$. This filter performs very well if one wants to preserve the shapes that are close to the reference sample for any size while simplifying the rest of the image. The original image in Fig. 2 is color, and its processing is based on a total ordering of the color vectors (by computation of the Mahalanobis distance [5] combined with a lexicographic cascade [10]), which makes the return to the color space easy [4]. The simplified color images are obtained by filtering the graylevel distance map.

\section{INTENSITY LEVEL ADAPTIVE OPERATORS}

We extend the concept of viscous operators to a general class of intensity level-adaptive operators. Consider the classic scheme of a building a level-invariant flat operator $\psi$ from its binary version:

$$
\psi(f)=\bigvee_{h} h \cdot \psi\left[\chi_{h}(f)\right]
$$

Now, if we vary the binary operator $\psi_{h}$ at each level, we can build a level-varying semi-flat operator

$$
\phi(f)=\bigvee_{h} h \cdot \psi_{h}\left[\chi_{h}(f)\right]
$$

provided that all $\psi_{h}$ are increasing operators. We give several generalized examples from connected operators. (Next, the level $h$ will assume only integer values.) (A) Intensity-adaptive Reconstruction filters: Consider a reconstruction opening $\rho^{-}(f \mid m)$, with marker $m$ and reference $f$. If we create $m$ from multiscale erosions and vary the erosion scale at each level (from a maximum $s$ to a minimum $s / M$ ), we obtain a 'viscous' reconstruction opening at scale $s$ :

$$
\rho_{s}^{v-}(f \mid m)=\bigvee_{h \geq 1}^{M} h \cdot \rho^{-}\left[\chi_{h}(f) \mid \chi_{s / h}(m)\right]
$$

(B) Volume opening: This was defined in [17] as an adaptive area opening $\gamma$ whose scale varied at level $h$ so that Volume $=h$. Area $\geq \lambda$. It can now be seen as an intensity-adaptive connected filter for which $\psi_{h}$ equals at each level an area filter $\alpha$ with parameter $\lambda / h$, where $\lambda$ is the scale of the volume opening

$$
\gamma_{\lambda}(f)=\bigvee_{h \geq 1}^{M} h \cdot \alpha_{\lambda / h}(f)
$$

(C) Viscous ASF: In (8) we defined a viscous reconstruction ASF of type 2, used in Fig. 2. Similarly, we can define viscous ASFs based on area open/closings. (For type 1 we should start with closings.)

\section{PDES FOR VISCOUS OPERATORS}

The multiscale dilation and erosion of an image $f: \mathbb{R}^{2} \rightarrow \mathbb{R}$ by a unit disk $B$ as the scale-space functions

$$
\delta(x, y, t) \triangleq(f \oplus t B)(x, y), \quad \varepsilon(x, y, t) \triangleq(f \ominus t B)(x, y)
$$

where $\delta(x, y, 0)=\varepsilon(x, y, 0)=f(x, y)$. Then, the PDEs generating the multiscale flat dilations and erosions of $f$ are

$$
\partial_{t} \delta=\|\nabla \delta\|, \quad \partial_{t} \varepsilon=-\|\nabla \varepsilon\|,
$$

where $\|\cdot\|$ is Euclidean norm. (For more general shapes $B$ and norms see $[3,8]$.) For 1D images, the disk becomes the interval 


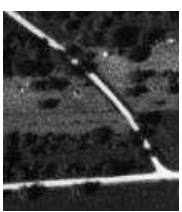

(a)

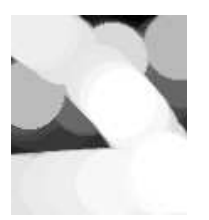

(b)

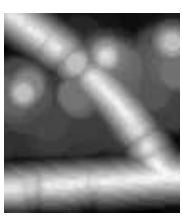

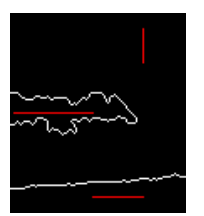

(c)

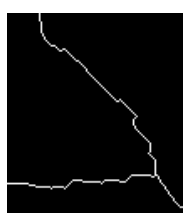

(d)

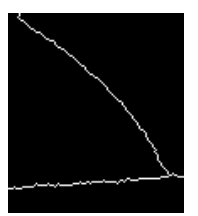

(e)

(f)

Fig. 1. Connection of thin lines by viscous dilation. (a) Original image. (b) Result of a standard dilation. (c) Result of a viscous dilation $\delta^{v 1}$ (of same maximal size). (d),(e), (f) are the watershed lines [9] of (a), (b), (c).
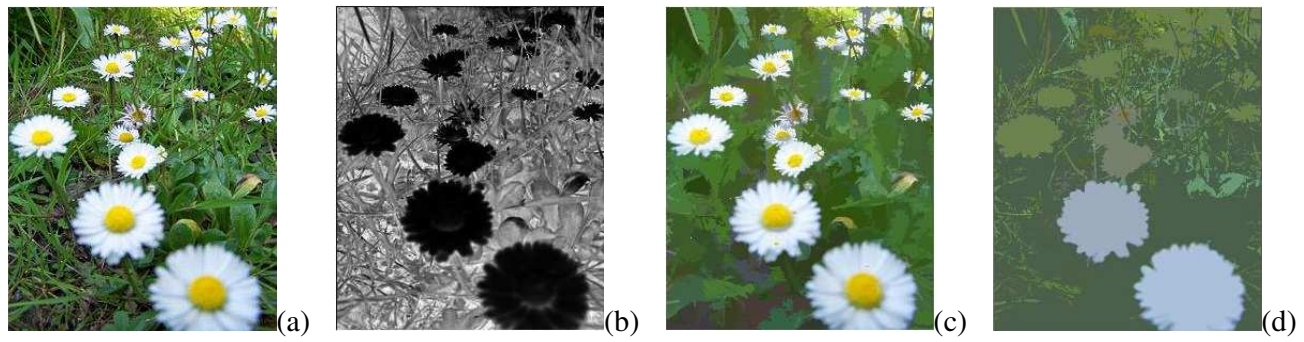

Fig. 2. Image simplification by viscous filtering. (a) Original image. (b) Mahalanobis distance map (the reference is a daisy). Viscous (c) and standard (d) alternating sequential filters by reconstruction (of same size).

$[-1,1]$, and we replace $\|\nabla u\|$ in all the above with $\left|\partial_{x} u\right|$. Examples of generating multiscale morphological operations by PDEs of the type $\partial u_{t}= \pm\left\|\partial_{x} u\right\|$ is shown in Fig. 3 (top).

The main objective is to find PDEs for the viscous dilations and erosion, since they are the building blocks of the rest viscous operators. We propose two models of PDEs for viscous dilations and erosions. Each model comes as a pair. First,

$$
\begin{aligned}
& \partial_{t} \delta^{v 1}(x, y, t)=\left(f_{\max }-\delta^{v 1}(x, y, t)\right)\left\|\nabla \delta_{1}^{v}\right\| \\
& \partial_{t} \varepsilon^{v 1}(x, y, t)=-\left(f_{\max }-\varepsilon^{v 1}(x, y, t)\right)\left\|\nabla \varepsilon_{1}^{v}\right\|
\end{aligned}
$$

where $f_{\text {max }}=\max _{x} f(x, y)$. To prove that they generate viscous operations, we use the fact from the level-set approach of [12] that the level curves of the function $\phi(x, y, t)$ that satisfies the PDE $\partial_{t} \phi=\beta(x, y, t)\|\nabla \phi\|$ move on the plane with normal speed $\beta(x, y, t)$. Thus, the isoheight curve at level $h$ of $\delta_{1}^{v}$, which is the boundary of the level set $X_{h}$, moves with speed $f_{\max }-h$, which is equivalent to the first type of viscous dilation. One generalization of the above PDEs is to replace the monotonic speeds $\beta=f_{\max }-\delta$ with $\beta=\max (a-\delta, 0)$ for some constant $a$. By using as speed anchor the global $\min f_{\min }$ of the initial function, we obtain another pair of viscous operations:

$$
\begin{aligned}
& \partial_{t} \delta^{v 2}(x, y, t)=\left(\delta^{v 2}-f_{\min }\right)\left\|\nabla \delta_{2}^{v}\right\| \\
& \partial_{t} \varepsilon^{v 2}(x, y, t)=-\left(\varepsilon^{v 2}-f_{\min }\right)\left\|\nabla \varepsilon_{2}^{v}\right\|
\end{aligned}
$$

In both models, the initial condition is the original image: $\delta^{v}(x, y, t)=$ $\varepsilon^{v}(x, y, t)=f(x, y)$. If $f \geq 0$, we may set $f_{\min }=0$.

\section{NUMERICAL ALGORITHMS FOR PDES}

The PDEs generating the flat or viscous dilations or erosions of $f$ by disks are special cases of Hamilton-Jacobi PDEs of the type $\phi_{t}+\beta\|\nabla \phi\|=0$, with initial condition $\phi(x, y, 0)=f(x, y)$ and speed $\beta= \pm\left(\phi_{\max }-\phi\right)$. In numerical simulations, the goal is to compute an approximation $\Phi_{i, j}^{n}$ of the true solution values $\phi(i \Delta x, j \Delta y, n \Delta t)$ on a grid with spatial steps $\Delta x, \Delta y$ and time (scale) step $\Delta t$, given the initial values $\Phi_{i, j}^{0}=f(i \Delta x, j \Delta y)$ and the velocities $\beta_{i j}=\beta(i \Delta x, j \Delta y)$. Such a first-order algorithm [12] replaces $\partial / \partial t$ with a forward difference and space derivatives with upwind combinations of finite differences

$$
\begin{aligned}
& D_{+x} \Phi_{i, j}^{n} \triangleq \frac{\Phi_{i+1, j}^{n}-\Phi_{i, j}^{n}}{\Delta x}, D_{-x} \Phi_{i, j}^{n} \triangleq \frac{\Phi_{i, j}^{n}-\Phi_{i-1, j}^{n}}{\Delta x_{n}^{n}} \\
& D_{+y} \Phi_{i, j}^{n} \triangleq \frac{\Phi_{i, j+1}^{n}-\Phi_{i, j}^{n}}{\Delta y}, D_{-y} \Phi_{i, j}^{n} \triangleq \frac{\Phi_{i, j}^{n}-\Phi_{i, j-1}^{n}}{\Delta y}
\end{aligned}
$$

and iterates the following:

$$
\Phi_{i, j}^{n+1}=\Phi_{i, j}^{n}-\Delta t\left[\left(\beta_{i j} \vee 0\right) \nabla^{+}+\left(\beta_{i j} \wedge 0\right) \nabla^{-}\right]
$$

where $n=0,1,2, \ldots,\left(T_{\max } / \Delta t\right), T_{\max }$ is the maximum time (or scale) of interest, and $\nabla^{+}, \nabla^{-}$are two different discretizations of the gradient magnitude required for positive and negative speed, respectively. One such discretization scheme was proposed in [12]. We use here an alternative discretization for the gradient magnitude results by replacing the partial derivative magnitudes $\left|\phi_{x}\right|,\left|\phi_{y}\right|$ in $\|\nabla \phi\|$ with the partial morphological derivatives $\mathcal{M}_{x}, \mathcal{M}_{y}$ and using the differences $D_{ \pm x}, D_{ \pm y}$ in place of the left/right derivatives required for $\mathcal{M}_{x}, \mathcal{M}_{y}$, as done in [7,8]:

$$
\begin{aligned}
\nabla_{M D}^{+} & =\left[\max \left(0, D_{-x},-D_{+x}\right)^{2}+\max \left(0, D_{-y},-D_{+y}\right)^{2}\right]^{1 / 2} \\
\nabla_{M D}^{+} & =\left[\min \left(0, D_{-x},-D_{+x}\right)^{2}+\min \left(0, D_{-y},-D_{+y}\right)^{2}\right]^{1 / 2}
\end{aligned}
$$

This $\mathcal{M}$-derivative discretization is less diffusive and faster than the gradient discretization of [12]. It is actually equivalent to but slighty faster than the gradient discretization proposed in [13] for solving the eikonal PDE. It is also interesting that the direct discretization of morphological derivatives yields a monotone and consistent numerical approximation to the Hamiltonian $\|\nabla \phi\|$.

The PDE approach provides a geometrically much better approximation to implementing multiscale morphological operations with Euclidean disks and hence avoid the abrupt shape discretization inherent in modeling digital dilations using discrete disks. Examples of generating multiscale viscous morphological operations 

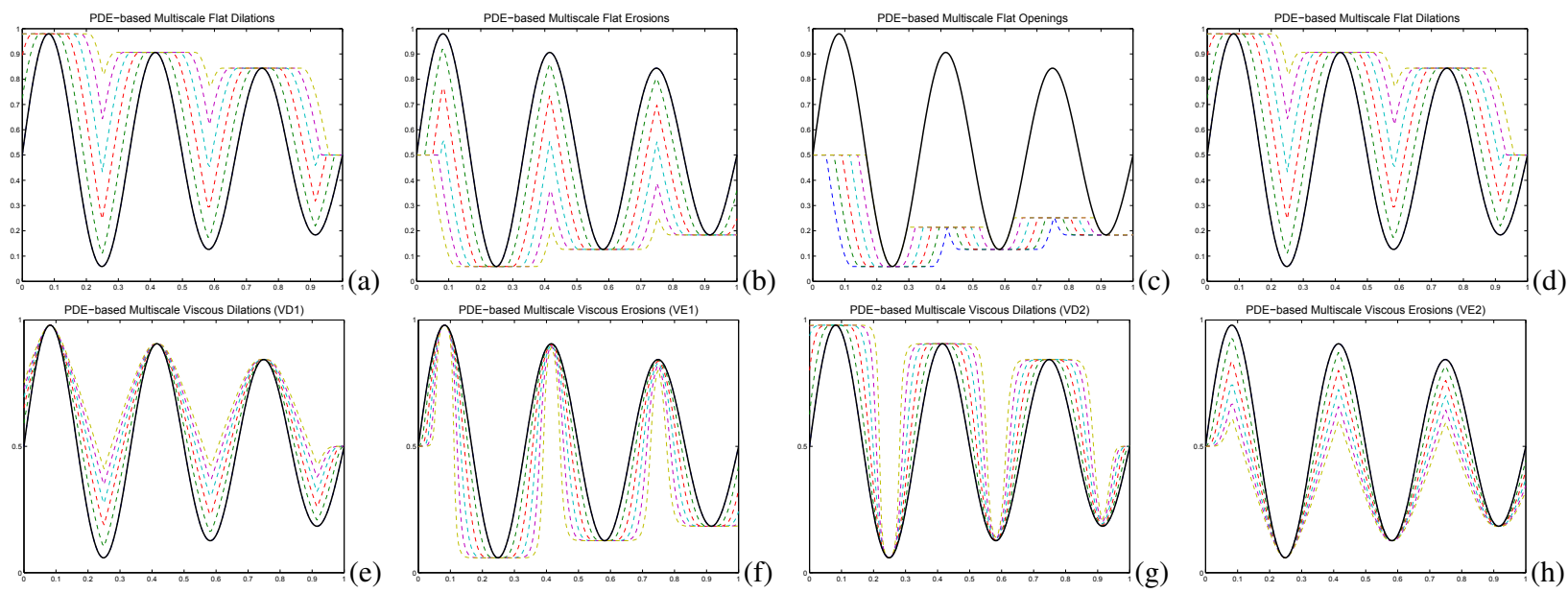

Fig. 3. Top: PDE-based evolutions (dash) of multiscale flat dilations, erosions, openings, and closings of a 1D signal $f$ (solid). Bottom: PDE-based evolutions of the two types of multiscale viscous dilations and erosions for same initial 1D signal $f$.

by PDEs of the type $\partial u_{t}= \pm\left(u_{\max }-u\right)\left\|\partial_{x} u\right\|$ and $\partial u_{t}= \pm(u-$ $\left.u_{\text {min }}\right)\left\|\partial_{x} u\right\|$ are shown in Fig. 3 (bottom).

\section{CONLUSIONS}

This paper generalizes the viscous operators of $[18,19]$ into a general class of intensity level adaptive operators, whose special cases include luminance-adaptive reconstruction and area filters as well as ASFs. Further, the paper introduces nonlinear scale-space PDEs that model these viscous operators and corresponding numerical algorithms that provide digital implementation with isotropy and increased speed (since images are not processed level by level). Image simulation examples demonstrate the utility of the viscous filters.

\section{REFERENCES}

[1] L. Alvarez, F. Guichard, P.L. Lions, and J-M. Morel, "Axioms and Fundamental Equations of Image Processing", Archiv. Rat. Mech., vol. 123 (3), pp. 199-257, 1993.

[2] A. Arehart, L. Vincent and B. Kimia, "Mathematical Morphology: The Hamilton-Jacobi Connection", in Proc. Int'l Conf. Comp. Vision, pp.215-219, 1993.

[3] R. Brockett and P. Maragos, "Evolution Equations for Continuous-Scale Morphological Filtering", IEEE Trans. Signal Processing, 42 (12): 3377-3386, 1994. Also, in Proc. ICASSP 1992.

[4] A. Garcia, Analyse Statistique et Morphologique des Images Multivaluées. Développements Logiciels pour les Applications Cliniques, PhD thesis, CMLA, ENS Cachan, June 2008.

[5] J. Goutsias, H.J.A.M. Heijmans and K. Sivakumar, "Morphological Operators for Image Sequences", Comp. Vision and Image Understanding, 62 (3): 326-346, 1995.

[6] F. Guichard, P. Maragos and J.-M. Morel, "Partial Differential Equations for Morphological Operators", in Space, Structure, and Randomness, edited by M. Bilodeau, F. Meyer and M. Schmitt, Springer Verlag, 2005, pp.369-390.

[7] P. Maragos, "Algebraic and PDE Approaches for Lattice ScaleSpaces with Global Constraints", Int'l J. Comp. Vision, vol.52 (2/3) pp.121-137, May 2003.
[8] P. Maragos, "Partial Differential Equations for Morphological Scale-Spaces and Eikonal Applications", The Image and Video Processing Handbook, 2nd Ed., A. Bovik, ed., Elsevier, 2005, pp.587-612.

[9] F. Meyer and S. Beucher, "Morphological segmentation," J. Visual Communication and Image Representation, vol.1, pp.2146, 1990.

[10] F. Meyer, "Grey-weighted, ultrametric and lexicographic distances", Proc. 7th Int'l Symp. Math. Morphology, Paris, 2005.

[11] F. Meyer and C. Vachier, "On the Regularization of The Watershed Transform", in Advances in Imaging and Electron Physics, Hawkes, P.W. (ed.), Acad. Press, 2007.

[12] S. Osher and J. Sethian, "Fronts Propagating with CurvatureDependent Speed: Algorithms Based on Hamilton-Jacobi Formulations", J. Comput. Physics, 79, pp.12-49, 1988.

[13] E. Rouy and A. Tourin, "A Viscocity Solutions Approach to Shape from Shading", SIAM J. Numer. Anal., vol.29 (3), pp.867-884, June 1992.

[14] G. Sapiro, R. Kimmel, D. Shaked, B. Kimia, and A. Bruckstein, "Implementing Continuous-scale Morphology via Curve Evolution”, Pattern Recognition, 26(9), pp.1363-1372, 1993.

[15] J. Serra, Image Analysis and Mathematical Morphology, Vol.2: Theoretical Advances, Acad. Press, 1988.

[16] J. Serra, "Viscous Lattices", J. Math. Imaging and Vision, 22(23): 269-282, 2005.

[17] A. Sofou and P. Maragos, "Generalized Flooding and Multicue PDE-based Image Segmentation", IEEE Trans. Image Processing, vol.17, no.3, pp.364-376, March 2008.

[18] C. Vachier and F. Meyer, "The Viscous Watershed Transform," J. Math. Imaging and Vision, vol.22, pp.251-267, 2005.

[19] C. Vachier and F. Meyer, "News from Viscous Land," Proc. 8th Int'l Symp. Math. Morphology, Rio de Janeiro, Brazil, 2007.

[20] R. van den Boomgaard and A. Smeulders, "The Morphological Structure of Images: The Differential Equations of Morphological Scale-Space", IEEE T-PAMI, 16: 1101-1113, 1994. 